\title{
The Impact of Financial Inclusion on Financial Inclusion through Social Empowerment through in Nanded District
}

\author{
Mudholkar Gajanan Panditrao, Rupali Gajanan Mudholkar
}

\begin{abstract}
In the present research work, the researcher tried to study the consequence of financial inclusion on social empowerment. The social empowerment is one of the essential indicators of human development. The social empowerment is achieved through its different parameter. The researcher made an attempt to analyze the relevance of financial inclusion in the context of social empowerment. For this purpose, researcher selected the sample size of $\mathbf{4 0 0}$ households both equally from rural and urban part of Nanded district. It is observed that there is significant difference in the level of social empowerment in rural and urban part of Nanded district. The findings, conclusions and suggestions are presented at the end of research paper.
\end{abstract}

Keywords : Social Empowerment and Financial Inclusion.

\section{INTRODUCTION}

\section{Financial Inclusion:}

According to RBI, Financial Inclusion is the process of ensuring access to appropriate financial products and services needed by vulnerable groups such as weaker sections and low income groups at an affordable cost in a fair and transparent manner by mainstream Institutional players.

According to World Bank report (2012), the objectives of financial inclusion can be accomplished by the strategies of financial inclusion. The strategies are the plans of actions defined to serve financial access to the underserved people.

According to Chakrabarty (2013) "Financial Inclusion is the process of ensuring access to appropriate financial products and services - deposit accounts, payment services, micro-credit and micro-level insurance - to vulnerable groups such as weaker sections and low income groups by mainstream institutional players."

\section{Social Empowerment}

Social empowerment is understood as the process of developing a sense of autonomy and self-confidence, and acting individually and collectively to change social relationships and the institutions and discourses that exclude poor people and keep them in poverty. Poor people's empowerment, and their ability to hold others to account, is strongly influenced by their individual assets (such as land,

Revised Manuscript Received on October 15, 2019.

* Correspondence Author

Dr. Mudholkar Gajanan Panditrao, Assistant Professor, SCMS, SRTM University, Nanded..

Mrs. Rupali Gajanan Mudholkar, Research Scholar, SCMS, SRTM University, Nanded.

housing, livestock, savings) and capabilities of all types: human (such as good health and education), social (such as social belonging, a sense of identity, leadership relations) and psychological (self-esteem, self-confidence, the ability to imagine and aspire to a better future). Also important are people's collective assets and capabilities, such as voice, organisation, representation and identity.

\section{OBJECTIVE OF RESEARCH STUDY}

To study the correlation between the social empowerment and financial inclusion in Nanded district.

\section{HYPOTHESIS OF RESEARCH STUDY}

The social empowerment and financial inclusion are not correlated in Nanded district before and after the financial inclusion.

\section{REVIEW OF LITERATURE}

According to Sharma \& Kukreja (2013), the impact of financial inclusion on socio-economic development was analyzed and it was found that the financial inclusion acts as a catalyst for the socio-economic development. The bank accounts are not opened in rural area it has to be raised in quantity and usage. The bank accounts and banking facility will empower the socio-economic development. There is scope for microscopic study.

According to Banerjee \&Francis (2014), the Social Development could be possible through the financial inclusion. The impact of financial inclusion on the social development was investigated by these researchers. The financial inclusion and human development index are directly correlated. The poverty alleviation and human development are made through the financial inclusion.

The human development index can be increased through the financial inclusion.

\section{RESEARCH METHODOLOGY}

The research methodology for the present research work is recited as follows.

1) Type of Research: Descriptive Research

2) Dependent Variable: Determinants of Social Empowerment

3) Independent Variables: The parameters of financial inclusion

4) Research Period: January to February 2017 
5) Methods of Data Collection: Interrogative Sample Survey Method

6) Universe: Users of Financial Inclusion Services in Nanded district.

7) Population: Users of Financial Inclusion in rural \& urban parts of Nanded district.

8) Sampling Frame: Rural and Urban Users of Financial Inclusion Equally.

9) Sample Size Determination: Using Mean Sampling Formula for large population.

10) Calculated Sample Size 400 respondents (200 rural and 200 urban)

11) Response Rate: $90 \%$ rural \& $90 \%$ urban respondents.

12) Sampling Method: Proportionate Stratified Sampling

13) Sources of Data Collection: Primary and Secondary sources.

14) Primary Sources: Structured questionnaire and observation.

15) Secondary sources: Journals, Articles, e resource databases

16) Measurement Scales: Nominal, ordinal and interval scale
17) Questions Types: Dichotomous, open ended, closed ended and ranking.

18) Rating Scales Used: Five Point Likert Scale.

19) Statistical Tools: $t$ test, ANOVA and descriptive statistical tools.

20) Statistical Software Package: Ms Excel 2010 and SPSS 20 .

\section{DATA ANALYSIS AND INTERPRETATION}

The impact of financial inclusion on social empowerment aspect of HDI is studied through following parameters as Social reputation has been raised due to FI, Your social status is positively affected (Buying behavior of customers to consumer durables), You participate in any social programs and festivals, Change in society is possible, Funding to the social programs and festivals is raised, Community-driven development is grown, Gender: social discrimination is reduced, Income generation may be same, Social accountability is more, Social cohesion is added, Social Inclusion is increased, Social Resilience \& Climate Change is enhanced, Social Sustainability \& Safeguards are raised, Overall, social empowerment is accomplished.

Table 1. Impact of FI on Social Empowerment Aspect of HDI

\begin{tabular}{|c|c|c|c|c|c|c|c|c|c|c|c|c|c|}
\hline & & \multicolumn{6}{|c|}{ Before } & \multicolumn{6}{|c|}{ After } \\
\hline $\begin{array}{c}\text { Sr.No } \\
\text {. }\end{array}$ & $\mathrm{T}$ & SD & D & $\mathrm{N}$ & A & SA & 홍 & SD & $\mathrm{D}$ & $\mathrm{N}$ & A & SA & है \\
\hline \multirow[t]{4}{*}{1.1} & $\mathrm{R}$ & 56 & 76 & 19 & 22 & 7 & 180 & 14 & 24 & 17 & 74 & 51 & 180 \\
\hline & $\%$ & 15.60 & 21.10 & 5.30 & 6.10 & 1.90 & 50 & 3.90 & 6.70 & 4.70 & 20.60 & 14.20 & 50 \\
\hline & $\mathrm{U}$ & 47 & 69 & 24 & 31 & 9 & 180 & 7 & 18 & 23 & 77 & 55 & 180 \\
\hline & $\%$ & 13.10 & 19.20 & 6.70 & 8.60 & 2.50 & 50 & 1.90 & 5.00 & 6.40 & 21.40 & 15.30 & 50 \\
\hline \multirow[t]{4}{*}{1.2} & $\mathrm{R}$ & 59 & 76 & 18 & 19 & 8 & 180 & 12 & 25 & 16 & 75 & 52 & 180 \\
\hline & $\%$ & 16.40 & 21.10 & 5.00 & 5.30 & 2.20 & 50 & 3.30 & 6.90 & 4.40 & 20.80 & 14.40 & 50 \\
\hline & $\mathrm{U}$ & 49 & 70 & 23 & 28 & 10 & 180 & 8 & 18 & 19 & 78 & 57 & 180 \\
\hline & $\%$ & 13.60 & 19.40 & 6.40 & 7.80 & 2.80 & 50 & 2.20 & 5.00 & 5.30 & 21.70 & 15.80 & 50 \\
\hline \multirow[t]{4}{*}{1.3} & $\mathrm{R}$ & 55 & 78 & 19 & 21 & 7 & 180 & 15 & 23 & 17 & 76 & 49 & 180 \\
\hline & $\%$ & 15.30 & 21.70 & 5.30 & 5.80 & 1.90 & 50 & 4.20 & 6.40 & 4.70 & 21.10 & 13.60 & 50 \\
\hline & $\mathrm{U}$ & 51 & 72 & 22 & 22 & 13 & 180 & 10 & 18 & 14 & 82 & 56 & 180 \\
\hline & $\%$ & 14.20 & 20.00 & 6.10 & 6.10 & 3.60 & 50 & 2.80 & 5.00 & 3.90 & 22.80 & 15.60 & 50 \\
\hline \multirow[t]{4}{*}{1.4} & $\mathrm{R}$ & 57 & 80 & 18 & 19 & 6 & 180 & 12 & 22 & 14 & 80 & 52 & 180 \\
\hline & $\%$ & 15.80 & 22.20 & 5.00 & 5.30 & 1.70 & 50 & 3.30 & 6.10 & 3.90 & 22.20 & 14.40 & 50 \\
\hline & $\mathrm{U}$ & 48 & 79 & 21 & 23 & 9 & 180 & 12 & 15 & 15 & 81 & 57 & 180 \\
\hline & $\%$ & 13.30 & 21.90 & 5.80 & 6.40 & 2.50 & 50 & 3.30 & 4.20 & 4.20 & 22.50 & 15.80 & 50 \\
\hline \multirow[t]{4}{*}{1.5} & $\mathrm{R}$ & 51 & 78 & 20 & 23 & 8 & 180 & 15 & 21 & 17 & 78 & 49 & 180 \\
\hline & $\%$ & 14.20 & 21.70 & 5.60 & 6.40 & 2.20 & 50 & 4.20 & 5.80 & 4.70 & 21.70 & 13.60 & 50 \\
\hline & $\mathrm{U}$ & 52 & 74 & 20 & 24 & 10 & 180 & 9 & 17 & 13 & 86 & 55 & 180 \\
\hline & $\%$ & 14.40 & 20.60 & 5.60 & 6.70 & 2.80 & 50 & 2.50 & 4.70 & 3.60 & 23.90 & 15.30 & 50 \\
\hline \multirow[t]{4}{*}{1.6} & $\mathrm{R}$ & 58 & 77 & 21 & 15 & 9 & 180 & 13 & 20 & 17 & 77 & 53 & 180 \\
\hline & $\%$ & 16.10 & 21.40 & 5.80 & 4.20 & 2.50 & 50 & 3.60 & 5.60 & 4.70 & 21.40 & 14.70 & 50 \\
\hline & $\mathrm{U}$ & 54 & 74 & 25 & 21 & 6 & 180 & 10 & 18 & 13 & 86 & 53 & 180 \\
\hline & $\%$ & 15.00 & 20.60 & 6.90 & 5.80 & 1.70 & 50 & 2.80 & 5.00 & 3.60 & 23.90 & 14.70 & 50 \\
\hline
\end{tabular}


International Journal of Recent Technology and Engineering (IJRTE) ISSN: 2277-3878, Volume-8, Issue-3S, October 2019

\begin{tabular}{|c|c|c|c|c|c|c|c|c|c|c|c|c|c|}
\hline 1.7 & $\mathrm{R}$ & 60 & 84 & 15 & 17 & 4 & 180 & 16 & 19 & 19 & 78 & 48 & 180 \\
\hline & $\%$ & 16.70 & 23.30 & 4.20 & 4.70 & 1.10 & $\mathbf{5 0}$ & 4.40 & 5.30 & 5.30 & 21.70 & 13.30 & 50 \\
\hline & $\mathrm{U}$ & 53 & 82 & 24 & 14 & 7 & 180 & 12 & 17 & 11 & 82 & 58 & 180 \\
\hline & $\%$ & 14.70 & 22.80 & 6.70 & 3.90 & 1.90 & $\mathbf{5 0}$ & 3.30 & 4.70 & 3.10 & 22.80 & 16.10 & $\mathbf{5 0}$ \\
\hline \multirow[t]{4}{*}{1.8} & $\mathrm{R}$ & 59 & 87 & 14 & 14 & 6 & 180 & 13 & 20 & 15 & 83 & 49 & 180 \\
\hline & $\%$ & 16.40 & 24.20 & 3.90 & 3.90 & 1.70 & $\mathbf{5 0}$ & 3.60 & 5.60 & 4.20 & 23.10 & 13.60 & $\mathbf{5 0}$ \\
\hline & $\mathrm{U}$ & 56 & 82 & 18 & 20 & 4 & 180 & 4 & 22 & 13 & 85 & 56 & 180 \\
\hline & $\%$ & 15.60 & 22.80 & 5.00 & 5.60 & 1.10 & 50 & 1.10 & 6.10 & 3.60 & 23.60 & 15.60 & 50 \\
\hline \multirow[t]{4}{*}{1.9} & $\mathrm{R}$ & 61 & 86 & 18 & 11 & 4 & 180 & 15 & 18 & 18 & 81 & 48 & 180 \\
\hline & $\%$ & 16.90 & 23.90 & 5.00 & 3.10 & 1.10 & $\mathbf{5 0}$ & 4.20 & 5.00 & 5.00 & 22.50 & 13.30 & $\mathbf{5 0}$ \\
\hline & $\mathrm{U}$ & 53 & 87 & 15 & 19 & 6 & 180 & 7 & 18 & 16 & 82 & 57 & 180 \\
\hline & $\%$ & 14.70 & 24.20 & 4.20 & 5.30 & 1.70 & 50 & 1.90 & 5.00 & 4.40 & 22.80 & 15.80 & $\mathbf{5 0}$ \\
\hline \multirow[t]{4}{*}{1.10} & $\mathrm{R}$ & 66 & 82 & 19 & 10 & 3 & 180 & 13 & 18 & 18 & 81 & 50 & 180 \\
\hline & $\%$ & 18.30 & 22.80 & 5.30 & 2.80 & 0.80 & $\mathbf{5 0}$ & 3.60 & 5.00 & 5.00 & 22.50 & 13.90 & $\mathbf{5 0}$ \\
\hline & $\mathrm{U}$ & 51 & 76 & 20 & 24 & 9 & 180 & 12 & 18 & 15 & 81 & 54 & 180 \\
\hline & $\%$ & 14.20 & 21.10 & 5.60 & 6.70 & 2.50 & $\mathbf{5 0}$ & 3.30 & 5.00 & 4.20 & 22.50 & 15.00 & $\mathbf{5 0}$ \\
\hline \multirow[t]{4}{*}{1.11} & $\mathrm{R}$ & 65 & 82 & 18 & 10 & 5 & 180 & 15 & 17 & 19 & 82 & 47 & 180 \\
\hline & $\%$ & 18.10 & 22.80 & 5.00 & 2.80 & 1.40 & $\mathbf{5 0}$ & 4.20 & 4.70 & 5.30 & 22.80 & 13.10 & $\mathbf{5 0}$ \\
\hline & $\mathrm{U}$ & 47 & 84 & 18 & 21 & 10 & 180 & 9 & 16 & 17 & 79 & 59 & 180 \\
\hline & $\%$ & 13.10 & 23.30 & 5.00 & 5.80 & 2.80 & $\mathbf{5 0}$ & 2.50 & 4.40 & 4.70 & 21.90 & 16.40 & $\mathbf{5 0}$ \\
\hline \multirow[t]{4}{*}{1.12} & $\mathrm{R}$ & 68 & 81 & 17 & 11 & 3 & 180 & 13 & 17 & 13 & 86 & 51 & 180 \\
\hline & $\%$ & 18.90 & 22.50 & 4.70 & 3.10 & 0.80 & 50 & 3.60 & 4.70 & 3.60 & 23.90 & 14.20 & 50 \\
\hline & $\mathrm{U}$ & 52 & 80 & 18 & 22 & 8 & 180 & 7 & 17 & 14 & 82 & 60 & 180 \\
\hline & $\%$ & 14.40 & 22.20 & 5.00 & 6.10 & 2.20 & $\mathbf{5 0}$ & 1.90 & 4.70 & 3.90 & 22.80 & 16.70 & $\mathbf{5 0}$ \\
\hline \multirow[t]{4}{*}{1.13} & $\mathrm{R}$ & 65 & 81 & 18 & 10 & 6 & 180 & 17 & 13 & 14 & 82 & 54 & 180 \\
\hline & $\%$ & 18.10 & 22.50 & 5.00 & 2.80 & 1.70 & $\mathbf{5 0}$ & 4.70 & 3.60 & 3.90 & 22.80 & 15.00 & $\mathbf{5 0}$ \\
\hline & $\mathrm{U}$ & 51 & 75 & 23 & 22 & 9 & 180 & 9 & 13 & 14 & 82 & 62 & 180 \\
\hline & $\%$ & 14.20 & 20.80 & 6.40 & 6.10 & 2.50 & $\mathbf{5 0}$ & 2.50 & 3.60 & 3.90 & 22.80 & 17.20 & $\mathbf{5 0}$ \\
\hline \multirow[t]{4}{*}{1.14} & $\mathrm{R}$ & 51 & 89 & 17 & 16 & 7 & 180 & 18 & 14 & 13 & 85 & 50 & 180 \\
\hline & $\%$ & 14.20 & 24.70 & 4.70 & 4.40 & 1.90 & $\mathbf{5 0}$ & 5.00 & $\mathbf{3 . 9 0}$ & 3.60 & 23.60 & 13.90 & $\mathbf{5 0}$ \\
\hline & $\mathrm{U}$ & 54 & 75 & 22 & 22 & 7 & 180 & 7 & 14 & 16 & 80 & 63 & 180 \\
\hline & $\%$ & 15.00 & 20.80 & 6.10 & 6.10 & 1.90 & $\mathbf{5 0}$ & 1.90 & 3.90 & 4.40 & 22.20 & 17.50 & $\mathbf{5 0}$ \\
\hline
\end{tabular}

Source: Field Survey and SPSS output

The Impact of FI on Social Empowerment Aspect of HDI is consolidated as follows

Table 2. Consolidation of Impact of FI on Social Empowerment

\begin{tabular}{|c|c|c|c|c|c|}
\hline & & $\mathrm{R}$ & $\%$ & $\mathrm{U}$ & $\%$ \\
\hline & SD & 51 to 68 & 14.2 to 18.9 & 47 to 56 & 13.1 to 15.6 \\
\hline Before & D & 76 to 89 & 21.2 to 24.8 & 69 to 87 & 19.2 to 24.2 \\
\hline & N & 14 to 21 & 3.9 to 5.9 & 15 to 25 & 4.2 to 7 \\
\hline & A & 10 to 23 & 2.8 to 6.4 & 14 to 31 & 3.9 to 8.7 \\
\hline & SA & 3 to 9 & 0.9 to 2.5 & 4 to 13 & 1.2 to 3.7 \\
\hline & Total & 180 & 50 & 180 & 50 \\
\hline & SD & 12 to 18 & 3.4 to 5 & 4 to 12 & 1.2 to 3.4 \\
\hline & D & 13 to 25 & 3.7 to 7 & 13 to 22 & 3.7 to 6.2 \\
\hline After & N & 13 to 19 & 3.7 to 5.3 & 11 to 19 & 3.1 to 5.3 \\
\hline & A & 74 to 86 & 20.6 to 23.9 & 77 to 86 & 21.4 to 23 \\
\hline & SA & 47 to 54 & 13.1 to 15 & 53 to 63 & 13.1 to 15 \\
\hline & Total & 180 & 50 & 180 & 50 \\
\hline
\end{tabular}

Source: Field Survey and SPSS output 
From the above table no. 2, it is apparent that 51 to 68 (14.2 to $18.9 \%$ ) and 47 to $56(13.1$ to $15.6 \%)$ rural and urban respondents strongly disagree that there is an impact of FI on social empowerment aspect of HDI before the financial inclusion respectively. Due to the prologue of financial inclusion policy, it is abridged to 12 to $18(3.4 \%$ to $5 \%)$ and 4 to $12(1.2 \%$ to $3.4 \%)$ rural and urban respondents strongly disagree the same after the financial inclusion. The 76 to 89 ( $21.2 \%$ to $24.8 \%$ ) and 69 to $87(19.2 \%$ to $24.2 \%)$ rural and urban respondents disagree that there is an impact of FI on social empowerment aspect of HDI before the financial inclusion respectively. Due to the prologue of financial inclusion policy, it is reduced to 13 to 25 ( 3.7 to $7 \%$ ) and 13 to $22(3.7 \%$ to $6.2 \%)$ rural and urban respondents disagree the same after the financial inclusion. The impact of neutral opinion before and after the financial inclusion is very negligible in rural and urban respondents.

The 10 to 23 (2.8 to $6.4 \%$ ) and 14 to $31(3.9$ to $8.7 \%$ ) rural and urban respondents agree that there is an impact of FI on social empowerment aspect of HDI before the financial inclusion respectively. Due to the preamble of financial inclusion policy, it is increased to 74 to 86 (20.6\% to $23.9 \%)$ and 77 to $86(21.4 \%$ to $23 \%)$ rural and urban respondents agree the same after the financial inclusion.

The 3 to 9 (0.9 to $2.5 \%)$ and 4 to $13(1.2$ to $3.7 \%)$ rural and urban respondents strongly agree that there is an impact of FI on social empowerment aspect of HDI before the financial inclusion respectively. Due to the beginning of financial inclusion policy, it is raised to 47 to 54 (13.1\% to $15 \%$ ) and 53 to 63 (13.1 to $15 \%$ ) rural and urban respondents strongly agree the same after the financial inclusion.

By using Test of Homogeneity of Variances (Levene Statistic), equality of variances are verified so as to know the heteroscedasticity and homoscedasticity It leads to the application of either $t$ test or ANOVA along with Robust Tests of Equality of Means as follows:

Table 3: Test of Homogeneity of Variances

\begin{tabular}{|c|c|c|c|c|c|}
\hline & $\begin{array}{l}\text { Ho: } \\
\text { Variances } \\
\text { are Equal }\end{array}$ & $\begin{array}{l}\text { Ho: Means } \\
\text { are Equal }\end{array}$ & $\begin{array}{c}\text { Test } \\
\text { Applicable }\end{array}$ & $\begin{array}{l}\text { Levene } \\
\text { Sign. }\end{array}$ & $\begin{array}{l}\text { Robust } \\
\text { Signi. }\end{array}$ \\
\hline $\begin{array}{l}\text { 1.1) Before-Social reputation has been } \\
\text { raised due to FI. }\end{array}$ & $\begin{array}{l}\text { Rejected } \\
\text { Unequal }\end{array}$ & $\begin{array}{l}\text { Accepted } \\
\text { Equal }\end{array}$ & $\mathrm{t}$ test & .044 & .082 \\
\hline $\begin{array}{l}\text { 1.2) Before-Your social status is } \\
\text { positively affected (Buying behavior of } \\
\text { customers to consumer durables) }\end{array}$ & $\begin{array}{l}\text { Rejected } \\
\text { Unequal }\end{array}$ & $\begin{array}{l}\text { Accepted } \\
\text { Equal }\end{array}$ & $\mathrm{t}$ test & .038 & .076 \\
\hline $\begin{array}{l}\text { 1.3) Before-You participate in any social } \\
\text { programs and festivals. }\end{array}$ & $\begin{array}{l}\text { Accepted } \\
\text { Equal }\end{array}$ & $\begin{array}{l}\text { Accepted } \\
\text { Equal }\end{array}$ & ANOVA & .057 & .219 \\
\hline 1.4) Before-Change in society is possible. & $\begin{array}{l}\text { Accepted } \\
\text { Equal }\end{array}$ & $\begin{array}{l}\text { Accepted } \\
\text { Equal }\end{array}$ & ANOVA & .120 & .166 \\
\hline $\begin{array}{l}\text { 1.5) Before-Funding to the social } \\
\text { programs and festivals is raised. }\end{array}$ & $\begin{array}{l}\text { Accepted } \\
\text { Equal }\end{array}$ & $\begin{array}{l}\text { Accepted } \\
\text { Equal }\end{array}$ & ANOVA & .431 & .748 \\
\hline $\begin{array}{l}\text { 1.6) Before-Community-driven } \\
\text { development is grown. }\end{array}$ & $\begin{array}{l}\text { Accepted } \\
\text { Equal }\end{array}$ & $\begin{array}{l}\text { Accepted } \\
\text { Equal }\end{array}$ & ANOVA & .653 & .597 \\
\hline $\begin{array}{l}\text { 1.7) Before-Gender: social } \\
\text { discrimination is reduced. }\end{array}$ & $\begin{array}{l}\text { Accepted } \\
\text { Equal }\end{array}$ & $\begin{array}{l}\text { Accepted } \\
\text { Equal }\end{array}$ & ANOVA & .295 & .327 \\
\hline $\begin{array}{l}\text { 1.8) Before-Gender: Income generation } \\
\text { may be same. }\end{array}$ & $\begin{array}{l}\text { Accepted } \\
\text { Equal }\end{array}$ & $\begin{array}{l}\text { Accepted } \\
\text { Equal }\end{array}$ & ANOVA & .322 & .502 \\
\hline $\begin{array}{l}\text { 1.9) Before-Social accountability is } \\
\text { more. }\end{array}$ & $\begin{array}{l}\text { Accepted } \\
\text { Equal }\end{array}$ & $\begin{array}{l}\text { Accepted } \\
\text { Equal }\end{array}$ & ANOVA & .180 & .154 \\
\hline 1.10) Before-Social cohesion is added. & $\begin{array}{l}\text { Rejected } \\
\text { Unequal }\end{array}$ & $\begin{array}{l}\text { Rejected } \\
\text { Unequal }\end{array}$ & $\mathrm{t}$ test & .000 & .002 \\
\hline $\begin{array}{l}\text { 1.11) Before-Social Inclusion is } \\
\text { increased. }\end{array}$ & $\begin{array}{l}\text { Rejected } \\
\text { Unequal }\end{array}$ & $\begin{array}{l}\text { Rejected } \\
\text { Unequal }\end{array}$ & $t$ test & .009 & .006 \\
\hline $\begin{array}{l}\text { 1.12) Before-Social Resilience \& Climate } \\
\text { Change is enhanced. }\end{array}$ & $\begin{array}{l}\text { Rejected } \\
\text { Unequal }\end{array}$ & $\begin{array}{l}\text { Rejected } \\
\text { Unequal }\end{array}$ & $\mathrm{t}$ test & .011 & .006 \\
\hline $\begin{array}{l}\text { 1.13) Before-Social Sustainability \& } \\
\text { Safeguards are raised. }\end{array}$ & $\begin{array}{l}\text { Rejected } \\
\text { Unequal }\end{array}$ & $\begin{array}{l}\text { Rejected } \\
\text { Unequal }\end{array}$ & $\mathrm{t}$ test & .004 & .011 \\
\hline $\begin{array}{l}\text { 1.14) Before-Overall, social } \\
\text { empowerment is accomplished. }\end{array}$ & $\begin{array}{l}\text { Accepted } \\
\text { Equal }\end{array}$ & $\begin{array}{l}\text { Accepted } \\
\text { Equal }\end{array}$ & ANOVA & .082 & .493 \\
\hline $\begin{array}{l}\text { 1.1) After- Social reputation has been } \\
\text { raised due to FI. }\end{array}$ & $\begin{array}{l}\text { Rejected } \\
\text { Unequal }\end{array}$ & $\begin{array}{l}\text { Accepted } \\
\text { Equal }\end{array}$ & $\mathrm{t}$ test & .015 & .160 \\
\hline $\begin{array}{l}\text { 1.2) After- Your social status is positively } \\
\text { affected (Buying behavior of customers to } \\
\text { consumer durables) }\end{array}$ & $\begin{array}{l}\text { Rejected } \\
\text { Unequal }\end{array}$ & $\begin{array}{l}\text { Accepted } \\
\text { Equal }\end{array}$ & $\mathrm{t}$ test & .047 & .203 \\
\hline
\end{tabular}




\begin{tabular}{|c|c|c|c|c|c|}
\hline $\begin{array}{c}\text { 1.3) After- You participate in any social } \\
\text { programs and festivals. }\end{array}$ & $\begin{array}{c}\text { Rejected } \\
\text { Unequal }\end{array}$ & $\begin{array}{c}\text { Accepted } \\
\text { Equal }\end{array}$ & t test & .029 & .120 \\
\hline 1.4) After- Change in society is possible. & $\begin{array}{c}\text { Accepted } \\
\text { Equal }\end{array}$ & $\begin{array}{c}\text { Accepted } \\
\text { Equal }\end{array}$ & ANOVA & .310 & .417 \\
\hline $\begin{array}{c}\text { 1.5) After- Funding to the social } \\
\text { programs and festivals is raised. }\end{array}$ & $\begin{array}{c}\text { Rejected } \\
\text { Unequal }\end{array}$ & $\begin{array}{c}\text { Accepted } \\
\text { Equal }\end{array}$ & t test & .013 & .103 \\
\hline $\begin{array}{c}\text { 1.6) After- Community-driven } \\
\text { development is grown. }\end{array}$ & $\begin{array}{c}\text { Accepted } \\
\text { Equal }\end{array}$ & $\begin{array}{c}\text { Accepted } \\
\text { Equal }\end{array}$ & ANOVA & .127 & .440 \\
\hline $\begin{array}{c}\text { 1.7) After- Gender: social discrimination } \\
\text { is reduced. }\end{array}$ & $\begin{array}{c}\text { Accepted } \\
\text { Equal }\end{array}$ & $\begin{array}{c}\text { Accepted } \\
\text { Equal }\end{array}$ & ANOVA & .100 & .135 \\
\hline 1.8) After- Gender: Income generation & $\begin{array}{c}\text { Rejected } \\
\text { Unay be same. }\end{array}$ & $\begin{array}{c}\text { Accepted } \\
\text { Equal }\end{array}$ & t test & .025 & .130 \\
\hline 1.9) After- Social accountability is more. & $\begin{array}{c}\text { Rejected } \\
\text { Unequal }\end{array}$ & $\begin{array}{c}\text { Accepted } \\
\text { Equal }\end{array}$ & t test & .033 & .106 \\
\hline 1.10) After- Social cohesion is added. & $\begin{array}{c}\text { Accepted } \\
\text { Equal }\end{array}$ & $\begin{array}{c}\text { Accepted } \\
\text { Equal }\end{array}$ & ANOVA & .723 & .653 \\
\hline $\begin{array}{c}\text { 1.11) After- Social Inclusion is } \\
\text { increased. }\end{array}$ & $\begin{array}{c}\text { Accepted } \\
\text { Equal }\end{array}$ & $\begin{array}{c}\text { Accepted } \\
\text { Equal }\end{array}$ & ANOVA & .108 & .120 \\
\hline 1.12) After- Social Resilience \& Climate \\
Change is enhanced. & $\begin{array}{c}\text { Accepted } \\
\text { Equal }\end{array}$ & $\begin{array}{c}\text { Accepted } \\
\text { Equal }\end{array}$ & ANOVA & .150 & .221 \\
\hline 1.13) After- Social Sustainability \& \\
Safeguards are raised. & $\begin{array}{c}\text { Rejected } \\
\text { Unequal }\end{array}$ & $\begin{array}{c}\text { Accepted } \\
\text { Equal }\end{array}$ & t test & .036 & .144 \\
\hline $\begin{array}{c}\text { 1.14) After- Overall, social } \\
\text { empowerment is accomplished. }\end{array}$ & $\begin{array}{c}\text { Rejected } \\
\text { Unequal }\end{array}$ & $\begin{array}{c}\text { Accepted } \\
\text { Equal }\end{array}$ & t test & .010 & .048 \\
\hline
\end{tabular}

Source: Field Survey and SPSS output

From table no. 3 , it is verified to use t test and ANOVA as follows

Table 4: Independent sample t test

\begin{tabular}{|c|c|c|c|c|c|c|}
\hline & & $\mathrm{t}$ & Df & $\begin{array}{c}\text { Sig. } \\
\text { (2-tailed) }\end{array}$ & $\begin{array}{l}\text { Comparing } \\
\text { with } .05\end{array}$ & $\begin{array}{c}\text { Hypothesis } \\
\text { Decisionn }\end{array}$ \\
\hline \multirow{2}{*}{$\begin{array}{l}\text { 1.1) Before-Social } \\
\text { reputation has been raised } \\
\text { due to FI. }\end{array}$} & $\begin{array}{l}\text { Equal variances } \\
\text { assumed }\end{array}$ & $\begin{array}{c}-1.74 \\
2\end{array}$ & 358 & .082 & $\begin{array}{c}\text { Greater than } \\
.05\end{array}$ & Accepted \\
\hline & $\begin{array}{l}\text { Equal variances } \\
\text { not assumed }\end{array}$ & $\begin{array}{c}-1.74 \\
2\end{array}$ & $\begin{array}{c}356.55 \\
3\end{array}$ & .082 & $\begin{array}{c}\text { Greater than } \\
.05\end{array}$ & Accepted \\
\hline \multirow{2}{*}{$\begin{array}{l}\text { 1.2) Before-Your social } \\
\text { status is positively } \\
\text { affected (Buying behavior } \\
\text { of customers to consumer } \\
\text { durables) }\end{array}$} & $\begin{array}{l}\text { Equal variances } \\
\text { assumed }\end{array}$ & $\begin{array}{c}-1.78 \\
2\end{array}$ & 358 & .076 & $\begin{array}{c}\text { Greater than } \\
.05\end{array}$ & Accepted \\
\hline & $\begin{array}{l}\text { Equal variances } \\
\text { not assumed }\end{array}$ & $\begin{array}{c}-1.78 \\
2\end{array}$ & $\begin{array}{c}356.44 \\
8\end{array}$ & .076 & $\begin{array}{c}\text { Greater than } \\
\quad .05\end{array}$ & Accepted \\
\hline \multirow{2}{*}{$\begin{array}{l}\text { 1.10) Before-Social } \\
\text { cohesion is added. }\end{array}$} & $\begin{array}{c}\text { Equal variances } \\
\text { assumed }\end{array}$ & $\begin{array}{c}-3.14 \\
1\end{array}$ & 358 & .002 & Less than .05 & Rejected \\
\hline & $\begin{array}{l}\text { Equal variances } \\
\text { not assumed }\end{array}$ & $\begin{array}{c}-3.14 \\
1\end{array}$ & $\begin{array}{c}340.79 \\
9\end{array}$ & .002 & Less than .05 & Rejected \\
\hline \multirow{2}{*}{$\begin{array}{l}\text { 1.11) Before-Social } \\
\text { Inclusion is increased. }\end{array}$} & $\begin{array}{l}\text { Equal variances } \\
\text { assumed }\end{array}$ & $\begin{array}{c}-2.75 \\
6\end{array}$ & 358 & .006 & Less than .05 & Rejected \\
\hline & $\begin{array}{l}\text { Equal variances } \\
\text { not assumed }\end{array}$ & $\begin{array}{c}-2.75 \\
6 \\
\end{array}$ & $\begin{array}{c}349.55 \\
8 \\
\end{array}$ & .006 & Less than .05 & Rejected \\
\hline \multirow{2}{*}{$\begin{array}{l}\text { 1.12) Before-Social } \\
\text { Resilience \& Climate } \\
\text { Change is enhanced. }\end{array}$} & $\begin{array}{c}\text { Equal variances } \\
\text { assumed }\end{array}$ & $\begin{array}{c}-2.77 \\
2 \\
\end{array}$ & 358 & .006 & Less than .05 & Rejected \\
\hline & $\begin{array}{c}\text { Equal variances } \\
\text { not assumed }\end{array}$ & $\begin{array}{c}-2.77 \\
2 \\
\end{array}$ & $\begin{array}{c}346.15 \\
6 \\
\end{array}$ & .006 & Less than .05 & Rejected \\
\hline \multirow{2}{*}{$\begin{array}{c}\text { 1.13) Before-Social } \\
\text { Sustainability \& } \\
\text { Safeguards are raised. }\end{array}$} & $\begin{array}{c}\text { Equal variances } \\
\text { assumed }\end{array}$ & $\begin{array}{c}-2.56 \\
3 \\
\end{array}$ & 358 & .011 & Less than .05 & Rejected \\
\hline & $\begin{array}{c}\text { Equal variances } \\
\text { not assumed }\end{array}$ & $\begin{array}{c}-2.56 \\
3 \\
\end{array}$ & $\begin{array}{c}351.38 \\
0 \\
\end{array}$ & .011 & Less than .05 & Rejected \\
\hline \begin{tabular}{|c|}
$1.1)$ After- Social \\
reputation has been raised \\
\end{tabular} & $\begin{array}{c}\text { Equal variances } \\
\text { assumed }\end{array}$ & $\begin{array}{c}-1.40 \\
8 \\
\end{array}$ & 358 & .160 & $\begin{array}{c}\text { Greater than } \\
.05 \\
\end{array}$ & Accepted \\
\hline
\end{tabular}




\begin{tabular}{|c|c|c|c|c|c|c|}
\hline due to FI. & $\begin{array}{c}\text { Equal variances } \\
\text { not assumed }\end{array}$ & $\begin{array}{c}-1.40 \\
8\end{array}$ & $\begin{array}{c}351.98 \\
0\end{array}$ & .160 & $\begin{array}{l}\text { Greater than } \\
.05\end{array}$ & Accepted \\
\hline \multirow{2}{*}{$\begin{array}{l}\text { 1.2) After- Your social } \\
\text { status is positively } \\
\text { affected (Buying behavior } \\
\text { of customers to consumer } \\
\text { durables) }\end{array}$} & $\begin{array}{c}\text { Equal variances } \\
\text { assumed }\end{array}$ & $\begin{array}{c}-1.27 \\
5 \\
\end{array}$ & 358 & .203 & $\begin{array}{c}\text { Greater than } \\
.05\end{array}$ & Accepted \\
\hline & $\begin{array}{l}\text { Equal variances } \\
\text { not assumed }\end{array}$ & $\begin{array}{c}-1.27 \\
5\end{array}$ & $\begin{array}{c}354.90 \\
1\end{array}$ & .203 & $\begin{array}{l}\text { Greater than } \\
.05\end{array}$ & Accepted \\
\hline \multirow{2}{*}{$\begin{array}{l}\text { 1.3) After- You } \\
\text { participate in any social } \\
\text { programs and festivals. }\end{array}$} & $\begin{array}{c}\text { Equal variances } \\
\text { assumed }\end{array}$ & $\begin{array}{c}-1.55 \\
7\end{array}$ & 358 & .120 & $\begin{array}{c}\text { Greater than } \\
.05\end{array}$ & Accepted \\
\hline & $\begin{array}{c}\text { Equal variances } \\
\text { not assumed }\end{array}$ & $\begin{array}{c}-1.55 \\
7 \\
\end{array}$ & $\begin{array}{c}355.16 \\
9\end{array}$ & .120 & $\begin{array}{c}\text { Greater than } \\
.05\end{array}$ & Accepted \\
\hline \multirow{2}{*}{$\begin{array}{l}\text { 1.5) After- Funding to the } \\
\text { social programs and } \\
\text { festivals is raised. }\end{array}$} & $\begin{array}{c}\text { Equal variances } \\
\text { assumed }\end{array}$ & $\begin{array}{c}-1.63 \\
3 \\
\end{array}$ & 358 & .103 & $\begin{array}{c}\text { Greater than } \\
.05\end{array}$ & Accepted \\
\hline & $\begin{array}{c}\text { Equal variances } \\
\text { not assumed }\end{array}$ & $\begin{array}{c}-1.63 \\
3 \\
\end{array}$ & $\begin{array}{c}353.71 \\
2 \\
\end{array}$ & .103 & $\begin{array}{c}\text { Greater than } \\
.05\end{array}$ & Accepted \\
\hline \multirow{2}{*}{$\begin{array}{l}\text { 1.8) After- Gender: } \\
\text { Income generation may be } \\
\text { same. }\end{array}$} & $\begin{array}{c}\text { Equal variances } \\
\text { assumed }\end{array}$ & $\begin{array}{c}-1.51 \\
8\end{array}$ & 358 & .130 & $\begin{array}{c}\text { Greater than } \\
.05\end{array}$ & Accepted \\
\hline & $\begin{array}{c}\text { Equal variances } \\
\text { not assumed }\end{array}$ & $\begin{array}{c}-1.51 \\
8 \\
\end{array}$ & $\begin{array}{c}351.99 \\
9 \\
\end{array}$ & .130 & $\begin{array}{c}\text { Greater than } \\
.05 \\
\end{array}$ & Accepted \\
\hline \multirow{2}{*}{$\begin{array}{l}\text { 1.9) After- Social } \\
\text { accountability is more. }\end{array}$} & $\begin{array}{c}\text { Equal variances } \\
\text { assumed }\end{array}$ & $\begin{array}{c}-1.61 \\
8 \\
\end{array}$ & 358 & .106 & $\begin{array}{c}\text { Greater than } \\
.05 \\
\end{array}$ & Accepted \\
\hline & $\begin{array}{c}\text { Equal variances } \\
\text { not assumed }\end{array}$ & $\begin{array}{c}-1.61 \\
8 \\
\end{array}$ & $\begin{array}{c}353.58 \\
5 \\
\end{array}$ & .106 & $\begin{array}{c}\text { Greater than } \\
.05 \\
\end{array}$ & Accepted \\
\hline \multirow{2}{*}{$\begin{array}{c}\text { 1.13) After- Social } \\
\text { Sustainability \& } \\
\text { Safeguards are raised. }\end{array}$} & $\begin{array}{c}\text { Equal variances } \\
\text { assumed }\end{array}$ & $\begin{array}{c}-1.46 \\
5 \\
\end{array}$ & 358 & .144 & $\begin{array}{c}\text { Greater than } \\
.05\end{array}$ & Accepted \\
\hline & $\begin{array}{c}\text { Equal variances } \\
\text { not assumed }\end{array}$ & $\begin{array}{c}-1.46 \\
5 \\
\end{array}$ & $\begin{array}{c}352.98 \\
4 \\
\end{array}$ & .144 & $\begin{array}{c}\text { Greater than } \\
.05\end{array}$ & Accepted \\
\hline \multirow{2}{*}{$\begin{array}{l}\text { 1.14) After- Overall, } \\
\text { social empowerment is } \\
\text { accomplished. }\end{array}$} & $\begin{array}{c}\text { Equal variances } \\
\text { assumed }\end{array}$ & $\begin{array}{c}-1.98 \\
3 \\
\end{array}$ & 358 & .051 & $\begin{array}{c}\text { Greater than } \\
.05 \\
\end{array}$ & Accepted \\
\hline & $\begin{array}{l}\text { Equal variances } \\
\text { not assumed }\end{array}$ & $\begin{array}{c}-1.98 \\
3\end{array}$ & $\begin{array}{c}349.76 \\
4\end{array}$ & .051 & $\begin{array}{c}\text { Greater than } \\
.05\end{array}$ & Accepted \\
\hline
\end{tabular}

Source: Field Survey and SPSS output

Table 5: ANOVA

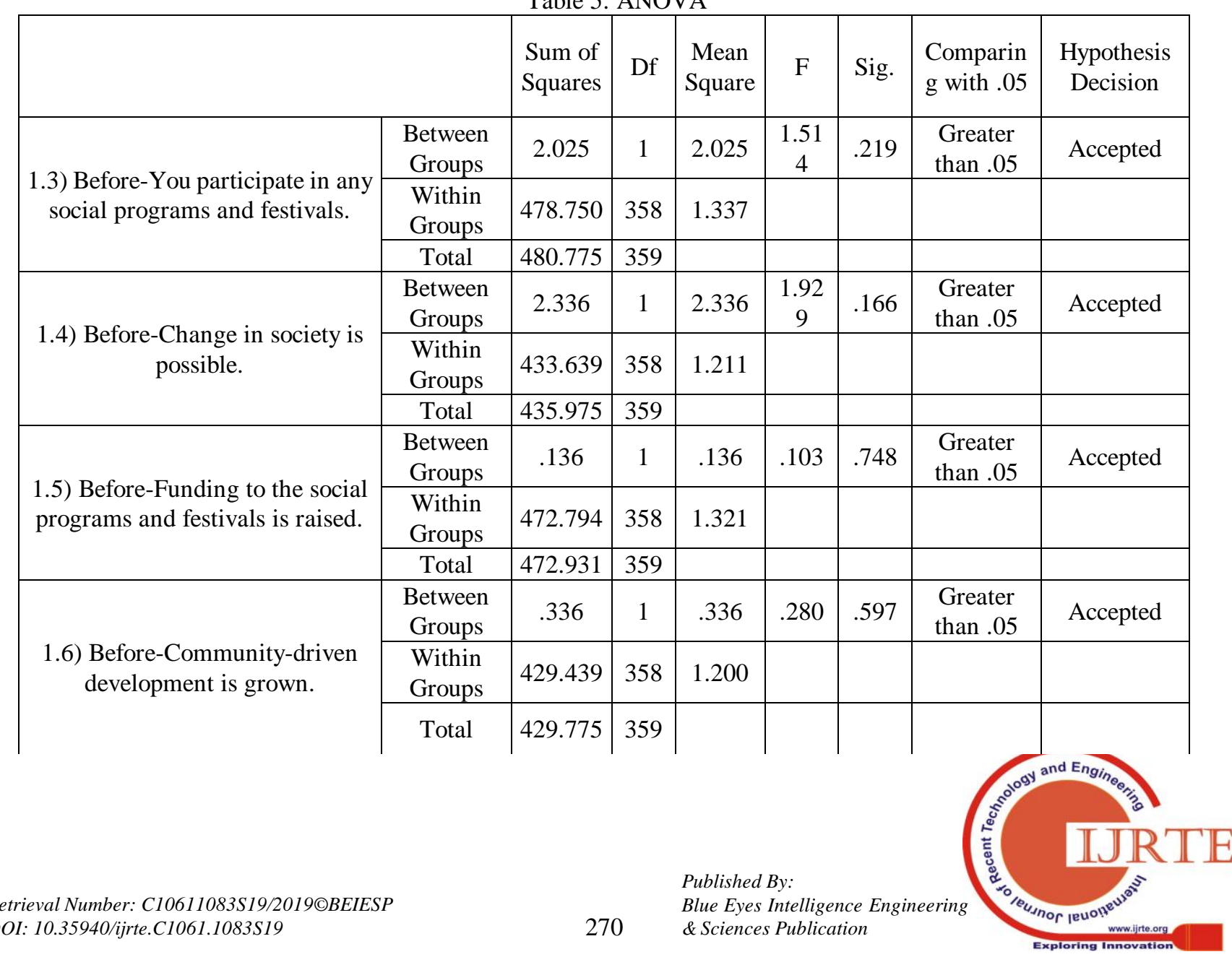




\begin{tabular}{|c|c|c|c|c|c|c|c|c|}
\hline \multirow{3}{*}{$\begin{array}{l}\text { 1.7) Before-Gender: social } \\
\text { discrimination is reduced. }\end{array}$} & $\begin{array}{c}\text { Between } \\
\text { Groups }\end{array}$ & 1.003 & 1 & 1.003 & .963 & .327 & $\begin{array}{l}\text { Greater } \\
\text { than } .05\end{array}$ & Accepted \\
\hline & $\begin{array}{l}\text { Within } \\
\text { Groups }\end{array}$ & 372.772 & 358 & 1.041 & & & & \\
\hline & Total & 373.775 & 359 & & & & & \\
\hline \multirow{3}{*}{$\begin{array}{l}\text { 1.8) Before-Gender: Income } \\
\text { generation may be same. }\end{array}$} & $\begin{array}{c}\text { Between } \\
\text { Groups }\end{array}$ & .469 & 1 & .469 & .452 & .502 & $\begin{array}{l}\text { Greater } \\
\text { than } .05\end{array}$ & Accepted \\
\hline & $\begin{array}{l}\text { Within } \\
\text { Groups }\end{array}$ & 371.906 & 358 & 1.039 & & & & \\
\hline & Total & 372.375 & 359 & & & & & \\
\hline \multirow{3}{*}{$\begin{array}{l}\text { 1.9) Before-Social accountability } \\
\text { is more. }\end{array}$} & $\begin{array}{c}\text { Between } \\
\text { Groups }\end{array}$ & 2.025 & 1 & 2.025 & $\begin{array}{c}2.04 \\
4\end{array}$ & .154 & $\begin{array}{l}\text { Greater } \\
\text { than } .05\end{array}$ & Accepted \\
\hline & $\begin{array}{l}\text { Within } \\
\text { Groups }\end{array}$ & 354.750 & 358 & .991 & & & & \\
\hline & Total & 356.775 & 359 & & & & & \\
\hline \multirow{3}{*}{$\begin{array}{l}\text { 1.14) Before-Overall, social } \\
\text { empowerment is accomplished. }\end{array}$} & $\begin{array}{c}\text { Between } \\
\text { Groups }\end{array}$ & .544 & 1 & .544 & .471 & .493 & $\begin{array}{l}\text { Greater } \\
\text { than } .05\end{array}$ & Accepted \\
\hline & $\begin{array}{l}\text { Within } \\
\text { Groups }\end{array}$ & 413.944 & 358 & 1.156 & & & & \\
\hline & Total & 414.489 & 359 & & & & & \\
\hline \multirow{3}{*}{$\begin{array}{l}\text { 1.4) After- Change in society is } \\
\text { possible. }\end{array}$} & $\begin{array}{c}\text { Between } \\
\text { Groups }\end{array}$ & .900 & 1 & .900 & .659 & .417 & $\begin{array}{l}\text { Greater } \\
\text { than } .05\end{array}$ & Accepted \\
\hline & $\begin{array}{l}\text { Within } \\
\text { Groups }\end{array}$ & 489.000 & 358 & 1.366 & & & & \\
\hline & Total & 489.900 & 359 & & & & & \\
\hline \multirow{3}{*}{$\begin{array}{l}\text { 1.6) After- Community-driven } \\
\text { development is grown. }\end{array}$} & $\begin{array}{c}\text { Between } \\
\text { Groups }\end{array}$ & .803 & 1 & .803 & .598 & .440 & $\begin{array}{l}\text { Greater } \\
\text { than } .05\end{array}$ & Accepted \\
\hline & $\begin{array}{l}\text { Within } \\
\text { Groups }\end{array}$ & 480.972 & 358 & 1.343 & & & & \\
\hline & Total & 481.775 & 359 & & & & & \\
\hline \multirow{3}{*}{$\begin{array}{l}\text { 1.7) After- Gender: social } \\
\text { discrimination is reduced. }\end{array}$} & $\begin{array}{c}\text { Between } \\
\text { Groups }\end{array}$ & 3.211 & 1 & 3.211 & $\begin{array}{c}2.25 \\
0\end{array}$ & .135 & $\begin{array}{l}\text { Greater } \\
\text { than } .05\end{array}$ & Accepted \\
\hline & $\begin{array}{l}\text { Within } \\
\text { Groups }\end{array}$ & 511.011 & 358 & 1.427 & & & & \\
\hline & Total & 514.222 & 359 & & & & & \\
\hline \multirow{3}{*}{$\begin{array}{l}\text { 1.10) After- Social cohesion is } \\
\text { added. }\end{array}$} & $\begin{array}{c}\text { Between } \\
\text { Groups }\end{array}$ & .278 & 1 & .278 & .203 & .653 & $\begin{array}{l}\text { Greater } \\
\text { than } .05\end{array}$ & Accepted \\
\hline & $\begin{array}{l}\text { Within } \\
\text { Groups }\end{array}$ & 489.678 & 358 & 1.368 & & & & \\
\hline & Total & 489.956 & 359 & & & & & \\
\hline \multirow{3}{*}{$\begin{array}{l}\text { 1.11) After- Social Inclusion is } \\
\text { increased. }\end{array}$} & $\begin{array}{c}\text { Between } \\
\text { Groups }\end{array}$ & 3.211 & 1 & 3.211 & $\begin{array}{c}2.42 \\
6 \\
\end{array}$ & .120 & $\begin{array}{l}\text { Greater } \\
\text { than } .05\end{array}$ & Accepted \\
\hline & $\begin{array}{l}\text { Within } \\
\text { Groups }\end{array}$ & 473.944 & 358 & 1.324 & & & & \\
\hline & Total & 477.156 & 359 & & & & & \\
\hline \multirow{3}{*}{$\begin{array}{l}\text { 1.12) After- Social Resilience \& } \\
\text { Climate Change is enhanced. }\end{array}$} & $\begin{array}{c}\text { Between } \\
\text { Groups }\end{array}$ & 1.878 & 1 & 1.878 & $\begin{array}{c}1.50 \\
5\end{array}$ & .221 & $\begin{array}{l}\text { Greater } \\
\text { than } .05\end{array}$ & Accepted \\
\hline & $\begin{array}{l}\text { Within } \\
\text { Groups }\end{array}$ & 446.744 & 358 & 1.248 & & & & \\
\hline & Total & 448.622 & 359 & & & & & \\
\hline
\end{tabular}

Source: Field Survey and SPSS output

From above table no. 4 and 5 , the hypothesis decisions are grouped as follows

\section{CONCLUSION}

The status of social empowerment's 14 parameters are studied before and after the financial inclusion and it is summarized as follows 
Table 6: Hypothesis 1.1-1.14 Decisions

\begin{tabular}{|c|c|c|c|}
\hline \multirow{2}{*}{$\begin{array}{l}\text { Sr. } \\
\text { No. }\end{array}$} & \multirow[t]{2}{*}{ Null Hypothesis: Title } & Before & After \\
\hline & & \multicolumn{2}{|c|}{ Financial Inclusion } \\
\hline 1.1 & Social reputation has been raised due to FI. & Accepted & Accepted \\
\hline 1.2 & $\begin{array}{l}\text { Your social status is positively affected (Buying behavior of } \\
\text { customers to consumer durables) }\end{array}$ & Accepted & Accepted \\
\hline 1.3 & You participate in any social programs and festivals. & Accepted & Accepted \\
\hline 1.4 & Change in society is possible. & Accepted & Accepted \\
\hline 1.5 & Funding to the social programs and festivals is raised. & Accepted & Accepted \\
\hline 1.6 & Community-driven development is grown. & Accepted & Accepted \\
\hline 1.7 & Gender: social discrimination is reduced. & Accepted & Accepted \\
\hline 1.8 & Gender: Income generation may be same. & Accepted & Accepted \\
\hline 1.9 & Social accountability is more. & Accepted & Accepted \\
\hline 1.10 & Social cohesion is added. & Rejected & Accepted \\
\hline 1.11 & Social Inclusion is increased. & Rejected & Accepted \\
\hline 1.12 & Social Resilience \& Climate Change is enhanced. & Rejected & Accepted \\
\hline 1.13 & Social Sustainability \& Safeguards are raised. & Rejected & Accepted \\
\hline 1.14 & Overall, social empowerment is accomplished. & Accepted & Accepted \\
\hline
\end{tabular}

Source: Field Survey and SPSS output

From the above 1.14 hypothesis decision, it is seen that the hypothesis 'the social empowerment and financial inclusion are not correlated in Nanded district before and after the financial inclusion' is accepted.

\section{REFERENCES}

1. Demirguc-Kunt, et. al. (2013). Measuring financial inclusion: Explaining variation in use of financial services across and within countries.

2. Government of India (2006), "Towards Faster and More Inclusive Growth: An Approach to the 11th Five Year Plan", Planning Commission, New Delhi, pp.93-99.

3. Government of India (2013). Report of the Committee for Evolving a Composite Development Index of States (Chairman: Dr. Raghuram G Rajan). Government of India: New Delhi.

4. Sarma, M. and J. Pais (2010). Financial Inclusion and Development. Journal of International Development 23, 613-628.

5. Sharma and Kukreja (2013), 'An analytical study: relevance of financial inclusion for developing nations. Research Inventy: International Journal of Engineering and Science, 2(6), Page No. 15-20.

6. Singh Kuldeep and Singh Kondan (2012) "Financial Inclusion, Development and Its Determinants: An Empirical Evidence of Indian States" The Asian Economic Review Vol.53.

7. Throat, U. (2007). "Financial Inclusion - The Indian Experience “, Speech by Smt. Usha Thorat, Deputy Governor, Reserve Bank of India.

8. Verma, Sakshi \& Aggarwal, Khushboo (2014). Financial inclusion through microfinance institutions in India. International Journal of Innovative Research \& Development, 3(1), 178-183.

9. World Bank (2005), Indicators of Financial Access - Household - Level Surveys

10. World Bank (2011-2015). Global Findex Database. The World Bank: Washington. 